\title{
Calculated Circular Dichroism of Acridine Orange Bound to Random-Coil Polypeptide
}

\author{
Toyoko IMAE \\ Department of Chemistry, Faculty of Science, Nagoya University, \\ Nagoya 464, Japan.
}

(Received March 2, 1977)

\begin{abstract}
A theory of circular dichroism for the amide transition of polypeptide in random-coil conformation has been developed on the basis of the exciton theory of optical activity and the Monte-Carlo approach to the treatment of statistical systems. This theory has further been extended in such a way as to be applicable for electronic transitions of chromophores bound to the side chains of the random-coil polypeptides. Circular dichroism is calculated for some binding models of acridine orange-randomcoil polypeptide complexes. The results are compared with those observed, and it is found that the longer axes of the bound dye molecules are almost parallel or perpendicular to a polypeptide chain, depending on whether ionization of the polypeptide is low or high.
\end{abstract}

KEY WORDS Circular Dichroism / Acridine Orange / RandomCoil Polypeptide / Poly $(S$-carboxymethyl-L-cysteine $)$ / Poly(L-glutamic acid) /

Recently we reported that various types of circular dichroism (CD) are induced on acridine orange bound to poly( $S$-carboxymethyl-L-cysteine) in solution. ${ }^{1}$ These are observed at different $\mathrm{pH}$ and $[\mathrm{P}] /[\mathrm{D}]$ (polymer residue/added dye) mixing ratios, and by polymers of different molecular weights. The observed $\mathrm{CD}$ is characteristic of the bonding species of the dye, dimeric dye or monomeric dye, as well as of the conformation of the polymer and can be classified into several types on the basis of the number and sign of the CD bands.

Type I CD is induced on an aggregate of dimeric dyes bound to a polymer in the $\beta$ conformation and has a pair of bands at 466 and $433 \mathrm{~nm}$. A model for the binding mode has been presented and discussed, ${ }^{2,3}$ in which the dimeric dye molecules attach to ionized carboxyl groups and stack together to form linear arrays on both sides of an extended polypeptide chain.

Types II and III CD have a pair of bands at 470 and $420 \mathrm{~nm}$ and additional bands at longer wavelength. These bands are opposite sign to each other for these two types. Types II $^{\prime}$ and III' $^{\prime} \mathrm{CD}$ have bands at 540 and $425 \mathrm{~nm}$ with opposite sign to each other. While Types II and II $^{\prime} \mathrm{CD}$ are exhibited by the solutions of random-coil polymers with low ionization, Types III and III' $^{\prime}$ CD are characteristic of highly ionized random-coil polymers and of solutions of low $[\mathrm{P}] /[\mathrm{D}]$ ratios.

The acridine orange-poly(L-glutamic acid) mixture also induces Types III and III $^{\prime} \mathrm{CD}$ at low $[\mathrm{P}] /[\mathrm{D}]$ ratios and alkaline $\mathrm{pH}^{4,5}$ The $\mathrm{CD}$ spectra similar to Types II and III were recently reported for an acridine orange-glycosaminoglycan mixture by Salter, et al., ${ }^{6}$ whose designation accidentally coincided with our's except for Type I.

Several theoretical investigations ${ }^{7-10}$ have attempted to evaluate the optical activity for the amide $\pi-\pi^{*}$ and $n-\pi^{*}$ transitions of polypeptide in random-coil conformation. However, these have relied on different approximations and methods and, consequently, have yielded inconsistent results. In the present work, we will apply a method of calculation to the optical 
activity of random-coil system, which was developed by means of an exciton theory of optical activity $^{11,12}$ and a Monte-Carlo approach for the treatment of statistical systems. Then we shall extend it to the case of the electronic transitions of dye chromophores bound to side chains of the random-coil polymer and discuss the binding models of acridine orange-random-coil polymer complexes which induce Types II, III, II', and III' $^{\prime}$ CD.

\section{CALCULATION}

\section{Binding Mode of Acridine Orange to the Random-} Coil Polypeptide

In solutions for which Type II CD is induced, carboxyl groups of the polymer are less ionized and adsorb dimeric dyes. ${ }^{1}$ The polymer chain should be rather compact and the bound dyes on it should form a short sequence. The polymer in the solutions inducing Type III CD is fully ionized and most of its carboxyl groups should be occupied by the dimeric cations. ${ }^{1}$ The high charge density and bulky bound dye should cause the polymer chain to extend, owing to electrostatic repulsion and steric hindrance of bound dyes. In Types II' and III' complexes, the binding unit is the monomeric dye, instead of the dimeric dye in Types II and III complexes.

Polarization of Absorption Bands

According to Zanker, ${ }^{13,14}$ the $492 \mathrm{~nm}$ absorption band of acridine orange can be assigned as the transition for the ${ }^{1} \mathrm{~L}_{\mathrm{b}}$ state and its polarization is along the longer axis of the dye molecular plane. The $470 \mathrm{~nm}$ band in the dimeric form, belonging to the same electronic transition as ${ }^{1} \mathrm{~L}_{\mathrm{b}}$, should have the same polarization as the monomeric form, when Zanker's antiparallel dimer model ${ }^{15}$ is assumed. The electrically forbidden ${ }^{1} \mathrm{~L}_{\mathrm{a}}$ transition of the monomeric dye may appear around $540 \mathrm{~nm}$ with a very small extinction coefficient, at the time the dye aggregates. ${ }^{13}$

The $260 \mathrm{~nm}$ band is assigned as the transition for the ${ }^{1} \mathbf{B}_{\mathrm{b}}$ state, ${ }^{16}$ whose polarization is along the longer axis of molecular plane, ${ }^{17}$ while the $290 \mathrm{~nm}$ band, assigned as the ${ }^{1} \mathbf{B}_{\mathrm{a}}$ transition, ${ }^{16}$ has a polarization parallel to the shorter axis, like the $540 \mathrm{~nm}$ band. ${ }^{17}$ Assignment of the band at $230 \mathrm{~nm}$ is not definite. Table I summarizes
Table I. Electronic parameters for the visible and ultraviolet transitions

\begin{tabular}{lccccc}
\hline $\begin{array}{l}\text { Bound } \\
\text { species }\end{array}$ & $\begin{array}{c}c / \nu_{\mathrm{E} 0}, \\
\mathrm{~nm}\end{array}$ & $\begin{array}{c}\mu_{0 \mathrm{e}}, \\
\mathrm{D}\end{array}$ & $\begin{array}{c}\Theta_{\mathrm{E}} / c, \\
\mathrm{~cm}^{-1}\end{array}$ & State & $\begin{array}{c}\text { Polarization } \\
\text { direction }\end{array}$ \\
\hline \multirow{4}{*}{ Dimer } & 540 & 1.00 & 1725 & ${ }^{1} \mathrm{~L}_{\mathrm{a}}$ & Shorter axis \\
& $458^{\mathrm{a}}$ & 9.18 & 1725 & ${ }^{1} \mathrm{~L}_{\mathrm{b}}$ & Longer axis \\
& 294 & $2.40^{\mathrm{b}}$ & - & ${ }^{1} \mathrm{~B}_{\mathrm{a}}$ & Shorter axis \\
& 261 & 10.29 & - & ${ }^{1} \mathrm{~B}_{\mathrm{b}}$ & Longer axis \\
& 230 & $2.50^{\mathrm{b}}$ & - & - & - \\
\hline \multirow{4}{*}{ Monomer } & 294 & 1.98 & - & ${ }^{1} \mathrm{~B}_{\mathrm{a}}$ & Shorter axis \\
& 261 & 8.43 & - & ${ }^{1} \mathrm{~B}_{\mathrm{b}}$ & Longer axis \\
& 230 & 2.06 & - & - & - \\
\hline
\end{tabular}

a Dimeric band of free acridine orange in $470 \mathrm{~nm}$ shifts to blue in the presence of polypeptide.

b These were calculated as $\mu_{0 \mathrm{e}}($ dimer $)=\left\{\mu_{0} \mathrm{~B}_{\mathrm{b}}\right.$ (dimer) $/$ $\mu_{0} 1_{\mathrm{B}}($ monomer $\left.)\right\} \mu_{0 \mathrm{e}}($ monomer $)=(10.29 / 8.43) \mu_{0 \mathrm{e}}$ (monomer).

these assignments.

We will focus our attention on calculation of conservative $C D$ for the ${ }^{1} L_{b}$ transition, since a pair of CD bands is observed on both sides of its absorption band. The ${ }^{1} \mathbf{B}_{\mathrm{b}}$ transition should make the largest contribution to the nonconservative term of $C D$ for the ${ }^{1} L_{b}$ transition, owing to its large transition dipole moment. For the electrically allowed ${ }^{1} L_{a},{ }^{1} L_{b},{ }^{1} B_{a}$, and ${ }^{1} B_{b}$ transitions, their magnetic moments are assumed to be small.

\section{Theory and Method}

The spatial configuration of the L-polypeptide chain is determined by the pairs of rotational angles, $\varphi$ around the $\mathrm{N}-\mathrm{C}^{\alpha}$ bond and $\phi$ around the $\mathrm{C}^{\alpha}-\mathrm{C}^{\prime}$ bond. We have adopted the convention in which bond rotation angles $\varphi$ and $\psi$ are zero in the planar trans conformation. ${ }^{18}$ The transformation matrix from the Cartesian reference frame of the residue $k+1$ to that of the residue $k$ is represented by $\boldsymbol{T}_{k}$ and is formulated as a function of $\varphi_{k}$ and $\psi_{k}$ associated with the residue $k .^{18}$ We consider that an array of dye formed along a random-coil polypeptide chain is composed of $N$ dye units attached to side chains of $N$ successive residues, numbered 1 to $N$, on a polymer chain. Thus the dye chromophores form an exciton, and an excited state $\mathrm{E}$ splits into $N$ exciton levels.

Let column vectors $\boldsymbol{\mu}_{k 0 \mathrm{e}}$ and $\boldsymbol{\mu}_{l 0 \mathrm{e}}$ be the electric 
transition dipole moments of the $k$-th and $l$-th chromophores, respectively, for the transition 0 -e. The rotatory strength for the $K$-th exciton level of an electrically allowed transition $0-\mathrm{E}$ is, to the zeroth order perturbation, expressed by,

$$
\begin{aligned}
R_{\mathrm{E} K}= & -\frac{\pi \nu_{\mathrm{E} 0}}{c} \sum_{k=1}^{N} \sum_{l>l e}^{N} C_{k \mathrm{e} K} C_{l \mathrm{e} K} \\
& \times\left\{\boldsymbol{r}_{k l} \cdot\left[\boldsymbol{\mu}_{k 0 \mathrm{e}} \times \boldsymbol{T}_{l l}^{\left({ }^{(l-k)}\right.} \boldsymbol{\mu}_{l 0 \mathrm{e}}\right]\right\}
\end{aligned}
$$

where $c$ is the light velocity, $\nu_{\mathrm{E} 0}$ the excitation frequency of a chromophore in the static field of the rest of the array, and $C_{k \mathrm{e} K}$ and $C_{l \mathrm{e} K}$ the exciton coefficients. $\quad \boldsymbol{r}_{k l}$ is the distance vector from the position of the transition dipole of the $k$-th chromophore to that of the $l$-th one. $\boldsymbol{T}_{k}^{(l-k)}$ means the serial product of $l-k$ factors, the first of which is $T_{k}$. The frequency for the $K$ th level of a transition $0-\mathrm{E}, \nu_{\mathrm{E} K}$, is shifted from the unpertubed frequency $\nu_{E 0}$ by the perturbation energy term $E_{\mathrm{E} K}^{\prime} / h$, where $h$ is Planck's constant. The exciton coefficients and the perturbation energies are obtained by the orthonormal conditions and by solving a set of applicable linear equations. ${ }^{3,12}$ The interaction potential $V_{k 0 \mathrm{e}, l 0 \mathrm{e}}$ between electric dipoles of the $k$-th and $l$-th chromophores, both for the transition $0-\mathrm{e}$, is described by the dipole approximation in the form

$$
\begin{aligned}
V_{k 00 \mathrm{e}, l 0 \mathrm{e}}= & \left\{\left(\boldsymbol{r}_{k l} \cdot \boldsymbol{r}_{k l}\right)\left(\boldsymbol{\mu}_{k 0 \mathrm{e}} \cdot \boldsymbol{T}_{k}^{(l-k)} \boldsymbol{\mu}_{l 0 \mathrm{e}}\right)\right. \\
& \left.-3\left(\boldsymbol{\mu}_{k 0 \mathrm{e}} \cdot \boldsymbol{r}_{k l}\right)\left(\boldsymbol{r}_{k l} \cdot \boldsymbol{T}_{k}^{(l-k)} \boldsymbol{\mu}_{l 0 \mathrm{e}}\right)\right\}\left(\boldsymbol{r}_{k l l} \cdot \boldsymbol{r}_{k l}\right)^{-5 / 2}
\end{aligned}
$$

where $V_{k 00, k 0 \mathrm{e}}=0$. The interaction potential matrix is symmetric. The molar ellipticity per chromophore associated with the excited state $\mathrm{E}$, at the frequency $\nu$, is written as

$$
[\theta]_{\mathrm{E}}=\frac{48 \pi^{2} N_{\mathrm{A}}}{h c N} \nu \sum_{K=1}^{N} R_{\mathrm{E} K} f\left(\nu-\nu_{\mathrm{E} K}\right)
$$

where $N_{\mathrm{A}}$ is Avogadro's number. $f\left(\nu-\nu_{\mathrm{E}_{K}}\right)$ is the shape function of a $\mathrm{CD}$ band associated with the level $\mathrm{E} K$ and is expressed as a Gaussian function with a half width $\Theta_{\mathrm{E}}$.

The first order CD of a transition 0-E arising from its electronic coupling with another electrically allowed transition $0-F$ is calculated by

$\sum_{X=1}^{N} R_{\mathrm{E} K}=-\sum_{K=1}^{N} R_{\mathrm{F} K}=\frac{2 \pi}{h c} \frac{\nu_{\mathrm{F} 0} \nu_{\mathrm{F} 0}}{\nu_{\mathrm{F} 0}^{2}-\nu_{\mathrm{E} 0}^{2}}$

$$
\begin{aligned}
& \times \sum_{k=1}^{N} \sum_{l>k}^{N}\left\{\left(\boldsymbol{r}_{k l} \cdot\left[\boldsymbol{\mu}_{k 0 \mathrm{e}} \times \boldsymbol{T}_{l k}^{(l-k)} \boldsymbol{\mu}_{l 0 \mathrm{f}}\right]\right) V_{k 0 \mathrm{e}, l 0 \mathrm{f}}\right. \\
& \left.+\left(\boldsymbol{r}_{k l} \cdot\left[\boldsymbol{\mu}_{k 0 \mathrm{f}} \times \boldsymbol{T}_{k}^{(l-k)} \boldsymbol{\mu}_{l 0 \mathrm{e}}\right]\right) V_{k 0 \mathrm{f}, l 0 \mathrm{e}}\right\} \\
& {[\theta]_{\mathrm{E}}=\frac{48 \pi^{2} N_{\mathrm{A}}}{h c N} \nu f\left(\nu-\nu_{\mathrm{E} 0}\right) \sum_{K=1}^{N} R_{\mathrm{E} K}}
\end{aligned}
$$

The interaction potentials $V_{k 0 \mathrm{e}, l 0 \mathrm{f}}$ and $V_{k 0 \mathrm{f}, l 0 \mathrm{e}}$ between different transitions $0-\mathrm{e}$ and $0-\mathrm{f}$ have forms identical with eq 2 , except for $\boldsymbol{\mu}_{l 0 \mathrm{f}}$ in place of $\boldsymbol{\mu}_{l 0 \mathrm{e}}$ or for $\boldsymbol{\mu}_{k 0 \mathrm{f}}$ in place of $\boldsymbol{\mu}_{k 0 \mathrm{e}}$. If the two excited states $E$ and $F$ have an identical polarization direction, eq 4 reduces to

$$
\begin{aligned}
\sum_{K^{\prime}=1}^{N} R_{\mathrm{E} K}= & -\sum_{K=1}^{N} R_{\mathrm{F} K}=\frac{4 \pi}{h c} \frac{\nu_{\mathrm{E} 0} \nu_{\mathrm{F} 0}}{\nu_{\mathrm{F} 0}^{2}-\nu_{\mathrm{E} 0}^{2}}\left(\frac{\mu_{0 \mathrm{f}}}{\mu_{0 \mathrm{e}}}\right)^{2} \\
& \times \sum_{k=1}^{N} \sum_{l>k}^{N}\left(\boldsymbol{r}_{k l} \cdot\left[\boldsymbol{\mu}_{k 0 \mathrm{e}} \times \boldsymbol{T}_{l l}^{(l-k)} \boldsymbol{\mu}_{l 0 \mathrm{e}}\right]\right) V_{l k 0 \mathrm{e}, l c \boldsymbol{\theta}}
\end{aligned}
$$

where $\mu_{0 \mathrm{e}}$ and $\mu_{0 \mathrm{f}}$ are the magnitudes of the dipole moments associated with the transitions $0-\mathrm{e}$ and $0-\mathrm{f}$.

The hypothetical virtual bonds which join successive $\alpha$-carbon atoms each other are represented by the bond vectors $l_{u, k}$ with a suffix $k$ numbered from 1 to $N$. The $X_{k}$ axis of the coordinate system affixed to bond $k$ is taken in the direction of bond $k$. The $Y_{k}$ axis is in the plane of bonds $k-1$ and $k$ with its positive direction chosen to render its projection on $X_{k-1}$ positive. The direction of the $Z_{k}$ axis completes a right-handed Cartesian coordinate system. Then, $l_{u, k}=l_{u}\left[\begin{array}{l}1 \\ 0 \\ 0\end{array}\right]$. The virtual bond length $l_{u}$ is taken as $3.80 \AA .^{18}$

Let $\boldsymbol{r}_{k}$ and $\boldsymbol{r}_{l}$ be the position column vectors for the transition moment in the $k$-th or $l$-th chromophore. Then,

$$
\boldsymbol{r}_{k l}=\sum_{i=1}^{l-k} \boldsymbol{T}_{k}^{(i)} \boldsymbol{l}_{u, k}+\boldsymbol{T}_{l}^{(l-k)} \boldsymbol{r}_{l}-\boldsymbol{r}_{k}
$$

It may be postulated that the direction of side chain is from $C^{\alpha}$ to $C^{\beta}$ and the transition moment of dye chromophore is situated on its extension. When $l_{\mathrm{c}}$ represents the distance between $\mathrm{C}^{\alpha}$ and the position of the transition moment, $\boldsymbol{r}_{k}$ is calculated to be 


$$
\begin{aligned}
\boldsymbol{r}_{k} & =\left[\begin{array}{l}
r_{k, X} \\
r_{k, Y} \\
r_{k, Z}
\end{array}\right] \\
& =l_{c}\left[\begin{array}{ccc}
\cos \xi & \sin \xi & 0 \\
-\sin \xi & \cos \xi & 0 \\
0 & 0 & 1
\end{array}\right]\left[\begin{array}{c}
\cos \theta^{\beta} \\
\sin \theta^{\beta} \cos \left(\varphi_{k}-120\right) \\
\sin \theta^{\beta} \sin \left(\varphi_{k}-120\right)
\end{array}\right]
\end{aligned}
$$

for an L-polypeptide. The angle $\xi$ between the virtual bond and the single bond $\mathrm{N}-\mathrm{C}^{\alpha}$ and the supplementary angle $\theta^{\beta}$ between $\mathrm{N}-\mathrm{C}^{\alpha}$ and $\mathrm{C}^{\alpha}-$ $\mathrm{C}^{\beta}$ are given as $13.2^{\circ}$ and $70.52^{\circ}$, respectively. ${ }^{18}$

We define the new coordinate system which describes the direction of electric transition dipole moment of the $k$-th dye chromophore. The $x_{k}$ axis is taken to be parallel to the direction from $\mathrm{C}_{k-1}^{\alpha}$ to $\mathrm{C}_{k+1}^{\alpha}$, and the $y_{k}$ axis is chosen to be the projection of the direction from $\mathrm{C}^{\alpha}$ to $\mathrm{C}^{\beta}$ on the plane perpendicular to the $x_{k}$ axis. The direction of the $z_{k}$ axis is determined so as to complete a right-handed Cartesian coordinate system.

The components of transition dipole moment vector which locates on the extension of the bond $\mathrm{C}_{k}^{\alpha}-\mathrm{C}_{k}^{\beta}$ can be represented by the angles $\theta$ and $\phi$ on the $x_{k} y_{k} z_{k}$ coordinate system at the position of the transition moment. $\theta$ is the angle between the $z_{k}$ axis and the dipole moment, and $\phi$ is the angle which the projection of dipole moment on the $x_{k} y_{k}$ plane makes with the $x_{k}$ axis. The components are transformed to that on the $X_{k} Y_{k} Z_{k}$ coordinate system as

$$
\begin{aligned}
& \boldsymbol{\mu}_{k 0 \mathrm{e}}=\left[\begin{array}{ccc}
a & b & a c \\
\sqrt{ } a^{2}+b^{2}+c^{2} & -\sqrt{ } a^{2}+b^{2} & -\sqrt{ } a^{2}+b^{2}+c^{2} \sqrt{ } a^{2}+b^{2} \\
b & a & b c \\
\sqrt{ } a^{2}+b^{2}+c^{2} & \sqrt{ } a^{2}+b^{2} & -\sqrt{ } a^{2}+b^{2}+c^{2} \sqrt{ } a^{2}+b^{2} \\
c & 0 & \sqrt{ } a^{2}+b^{2} \\
\sqrt{ } a^{2}+b^{2}+c^{2} & 0 & \sqrt{ } a^{2}+b^{2}+c^{2}
\end{array}\right]\left[\begin{array}{ccc}
1 & 0 & 0 \\
0 & d & e \\
\sqrt{ } d^{2}+e^{2} & \sqrt{ } d^{2}+e^{2} \\
0 & e & d \\
\sqrt{ } d^{2}+e^{2} & \sqrt{ } d^{2}+e^{2}
\end{array}\right]\left[\begin{array}{l}
\mu_{0 \mathrm{e}} \sin \theta \cos \phi \\
\mu_{0 \mathrm{e}} \sin \theta \sin \phi \\
\mu_{0 \mathrm{e}} \cos \theta
\end{array}\right] \\
& {\left[\begin{array}{l}
a \\
b \\
c
\end{array}\right]=\left(\boldsymbol{T}_{l i}+\boldsymbol{E}\right) \boldsymbol{l}_{u, k}} \\
& d=-\frac{1}{\sqrt{ } a^{2}+b^{2}}\left(b r_{k, X}-a r_{k, Y}\right) \\
& e=-\frac{1}{\sqrt{ } a^{2}+b^{2}+c^{2} \sqrt{ } a^{2}+b^{2}}\left\{a c r_{l, X}+b c r_{k, Y}-\left(a^{2}+b^{2}\right) r_{k, Z}\right\}
\end{aligned}
$$

where $\boldsymbol{E}$ is a unit matrix.

The five different regions of the angles $\varphi$ and $\psi$ were chosen. They were designated by Zubkov, et al..$^{9}$ as $\alpha_{1}\left(80^{\circ} \leq \varphi \leq 140^{\circ}, 100^{\circ} \leq \varphi \leq 140^{\circ}\right), \beta_{2}\left(0^{\circ} \leq\right.$ $\left.\varphi \leq 120^{\circ},-90^{\circ} \leq \psi \leq 0^{\circ}\right), \beta_{3}\left(30^{\circ} \leq \varphi \leq 90^{\circ},-90^{\circ} \leq\right.$ $\left.\psi \leq-30^{\circ}\right), \quad 1\left(0^{\circ} \leq \varphi \leq 150^{\circ},-210^{\circ} \leq \psi \leq 0^{\circ}\right)$, and $2\left(0^{\circ} \leq \varphi \leq 120^{\circ},-60^{\circ} \leq \psi \leq 0^{\circ}\right)$, and each of these was assumed to be a rectangular well. Angles were taken at $10^{\circ}$ intervals. The 50 random chains consisting of 10 residues $(M=50, N=10)$ were generated by choosing the pairs of angles $\varphi$ and $\psi$ at random from the allowed regions. The CD of 50 chains was averaged with the statistical weight of unity for all chains. The molar ellipticity calculated on the basic of mole of dimeric dye unit had to be divided by a factor 2 for comparison with those observed, because the observed value is based on the mole of monomeric dye.

If all carboxyl groups were occupied by the dye molecules, two dye molecules bound close to each other should undergo steric hindrance. Hence, when a dye with an excluded volume overlaps with the other dye, one of them must be removed; it was assumed that such dye can not bind to the carboxyl group with a large numbering. Thus the number of dyes bound to the $N$ successive amino acid residues becomes less than $N$ and it was designated as $N^{\prime}$. In the present case, the dye molecule has $9.0 \AA$ in length, $3.0 \AA$ in width and $3.0 \AA$ thick. Its 
thickness increases to $5.0 \AA$, when it dimerizes.

\section{Parameters}

The electronic parameters are given in Table I. The magnitudes of the electric transition dipole moments for monomer species were estimated graphically by the previously reported method $^{3}$ from the observed absorption spectrum of free acridine orange at $[\mathrm{D}]=5.0 \times 10^{-5} \mathrm{M}$ and

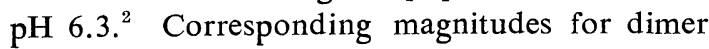
species were calculated from the observed spectrum of dye-poly(S-carboxymethyl-L-cysteine) mixture for $[\mathrm{P}] /[\mathrm{D}] 167$ at $[\mathrm{D}]=5.0 \times 10^{-5} \mathrm{M}$ and $\mathrm{pH} \mathrm{7.2.{ } ^ { 2 }}$ The half width of the visible bands was taken as $1725 \mathrm{~cm}^{-1}$, which was the same as that adopted previously. ${ }^{3}$

The distance $l_{\mathrm{c}}$ between $\mathrm{C}^{\alpha}$ and the position of transition moment of acridine orange was assumed to be $5.0 \AA$, approximately equal to the distance for the planar trans conformation of the side chain. The longer axis of each adsorbed dye molecule, i.e., the ${ }^{1} \mathrm{~L}_{\mathrm{b}}$ electric transition moment, was put at $\phi_{1}=0^{\circ}$, where the suffix $l$ refers to the longer axis of dye molecule.

For the system of dye- $\beta$ form poly $(S$-carboxymethyl-L-cysteine), i.e., for the two-fold helix, it is found that the parameters $\theta_{1}=10^{\circ}, \varphi=41^{\circ}$ and $\psi=315^{\circ 19}$ correspond to the parameters adopted in the helical parameter method. ${ }^{3}$ The $\mathrm{CD}$ bands calculated for $N=10$ by both methods were in relatively close agreement with each other.

\section{RESULTS}

\section{Amide $\pi-\pi^{*}$ Transition of Random-Coil Poly- peptide Chain}

The CD calculation of the random-coil polypeptide chain was carried out for the electrically allowed amide $\pi-\pi^{*}\left(c / \nu_{E 0}=190 \mathrm{~nm}\right)$ transition by the method given in Appendix. We attempted four kinds of computations for population-averaged $\mathrm{CD}$ spectra. In the first case, pairs of rotational angles $\varphi_{k}$ and $\phi_{k}$ were selected at random from the chosen region $\{\varphi, \phi\}$ and a chain composed of $N$ residues was generated. In this way $M$ chains were constructed. The CD was calculated for each of them and its statistical average with equal weight was computed. In the second, the $\mathrm{CD}$ of the chains generated in the same way as above was averaged with the statistical weight $\exp \left\{-\sum_{k=1}^{N-1} E\left(\varphi_{k}, \varphi_{k}\right) / R T\right\}$, where $E$ is the conformational energy, $R$ is gas constant and $T$ is the temperature. In the third, the angles $\varphi_{k}$ and $\phi_{k}$ were selected from the chosen region $\{\varphi, \psi\}$ with the weight given by conformational energy $\exp \{-E(\varphi, \psi) / R T\}$ and the $C D$ was averaged with an equal weight for all chains.

We chose the region of energy lower than $6 \mathrm{kcal} \mathrm{mol}^{-1}$ from the conformational energy map given by Brant, et al. ${ }^{20}$ The temperature $T$ was $293 \mathrm{~K}$. The convergence for $N=10$ was reached at a value for $M$ much less than 50 . The final results of all methods above gave similar spectra, having a positive $C D$ band around $198 \mathrm{~nm}$ and a negative $\mathrm{CD}$ band around $180 \mathrm{~nm}$. The calculation by the first method yielded a pair of bands with a molar ellipticity of 8,000 and $-9,000$ at most, which were much smaller than the observed values. ${ }^{21}$ The molar ellipticity calculated by the second method was smaller than that found by the first. For the third method, the calculated molar ellipticity was 27,000 at a longer wavelength side and $-31,000$ at a shorter wavelength side, in agreement with the observed values. In the 900 pairs of rotational angles $\varphi$ and $\psi$ selected by the third method, $81 \%$ was generated from the region with the lowest energy value of $E=0 \mathrm{kcal}$ $\mathrm{mol}^{-1}$, in spite of the fact that the region of $E=0 \mathrm{kcal} \mathrm{mol}^{-1}$ occupies, at most, $17 \%$ of all the region.

In the last method, the $\varphi$ and $\phi$ were generated from the region of $10^{\circ} \leq \varphi \leq 110^{\circ}$ and $280^{\circ} \leq \psi \leq$ $350^{\circ}$. An equal weight was assigned for the chosen region $\{\varphi, \psi\}$, although its region consisted of $75 \%$ of the region of $E=0 \mathrm{kcal} \mathrm{mol}^{-1}$ and $25 \%$ of the region of $E=1 \mathrm{kcal} \mathrm{mol}^{-1}$. The averaged $C D$ was calculated with an equal weight for all chains. The result was almost the same as that by the third method and therefore this method is used for the CD calculation of acridine orange-random-coil polymer mixture, for simplicity.

The ${ }^{1} L_{\mathrm{b}}$ Transition of Acridine Orange Bound to Random-Coil Polypeptide Chain

In Table II, the average end-to-end distance between $\mathrm{C}_{0}^{\alpha}$ and $\mathrm{C}_{\mathrm{N}}^{\alpha},\left\langle\mathrm{R}_{0 \mathrm{~N}}^{2}\right\rangle^{1 / 2}$, at $N=10$ is shown 
for each region. The end-to-end distances were in the following order: $\beta_{3}>2>\beta_{2} \alpha_{1}\left(P_{\alpha}=0.21\right)>$ $1>\beta_{2} \alpha_{1}\left(P_{\alpha}=0.57\right)>\alpha_{1}$, where $P_{\alpha}$ is a probability

Table II. End-to-end distance of random-coil polypeptide and $\left\langle N^{\prime}\right\rangle$ value for dimeric dyes bound to polypeptides $(M=50, N=10$, $\phi_{1}=0^{\circ}, \theta_{\mathrm{S}}=90^{\circ}, \phi_{\mathrm{s}}=90^{\circ}$, and $l_{\mathrm{c}}=5.0 \AA$ )

\begin{tabular}{lrcr}
\hline \multicolumn{1}{c}{ Region } & $\left\langle\mathrm{R}_{0 N}^{2}\right\rangle^{1 / 2}, \AA$ & \multicolumn{2}{c}{$\left\langle N^{\prime}\right\rangle$} \\
\hline$\beta_{3}$ & 31.29 & 6.3 & 8.4 \\
2 & 30.40 & 7.5 & 8.8 \\
$\beta_{2} \alpha_{1}\left(P_{\alpha}=0.21\right)$ & 22.30 & 7.0 & 7.7 \\
1 & 20.41 & 6.9 & 7.3 \\
$\beta_{2} \alpha_{1}\left(P_{c}=0.57\right)$ & 12.91 & 6.0 & 6.6 \\
$\alpha_{1}$ & 9.30 & 5.5 & 6.1 \\
\hline
\end{tabular}

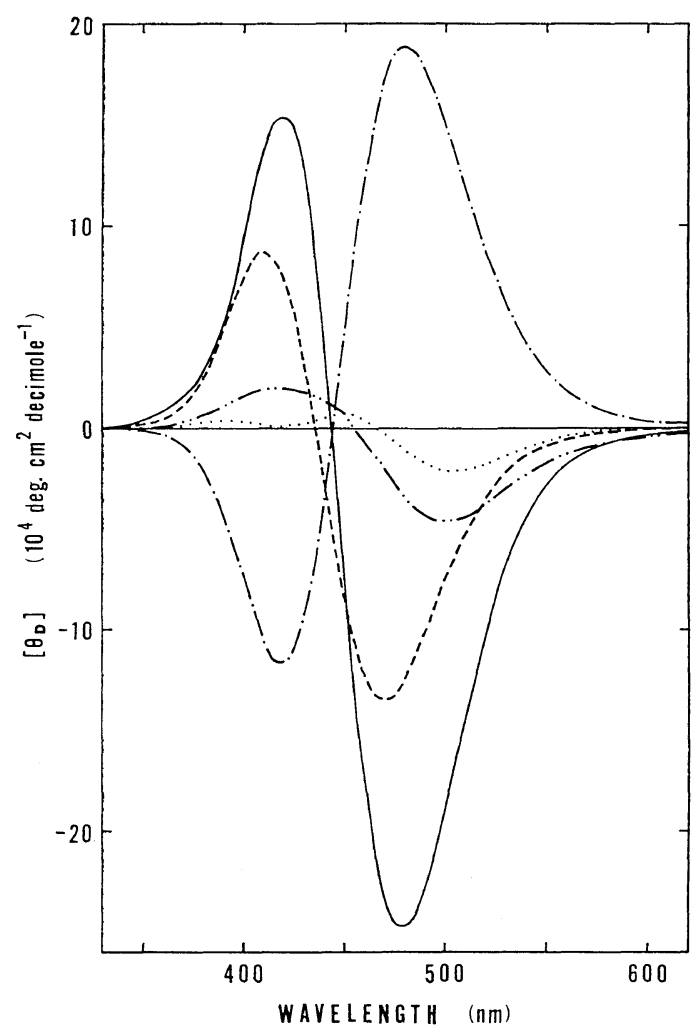

Figure 1. The $\theta_{1}$ dependence of the $C D$ of the ${ }^{1} \mathrm{~L}_{\mathrm{b}}$ transition calculated for dimeric dye bound to polypeptides generated from the $\alpha_{1}$ region: $-\cdot-, 0^{\circ} ; \cdots, 45^{\circ} ;-\cdots-, 90^{\circ} ;-, 115^{\circ}$; --$135^{\circ} ; M=50, N=10, \phi_{1}=0^{\circ}, \theta_{\mathrm{s}}=90^{\circ}, \phi_{\mathrm{s}}=90^{\circ}$, and $l_{\mathrm{c}}=5.0 \AA$. of the $\alpha$ region and $P_{\beta}=1-P_{\alpha}$. It may be noted that the end-to-end distance for the $\beta_{3}$ region is shorter than that of the antiparallel $\beta$-form and that the distance for the $\beta_{2} \alpha_{1}\left(P_{\alpha}=0.57\right)$ region is slightly shorter than that of the $\alpha$-helix.

The shorter axis of the dye was put parallel to the $y$ axis, that is, $\theta_{\mathrm{S}}=90^{\circ}$ and $\phi_{\mathrm{S}}=90^{\circ}$, where the suffix $s$ refers to the shorter axis of dye molecule. The $\left\langle N^{\prime}\right\rangle$ values for dimeric dyes bound to random-coil polypeptides are shown

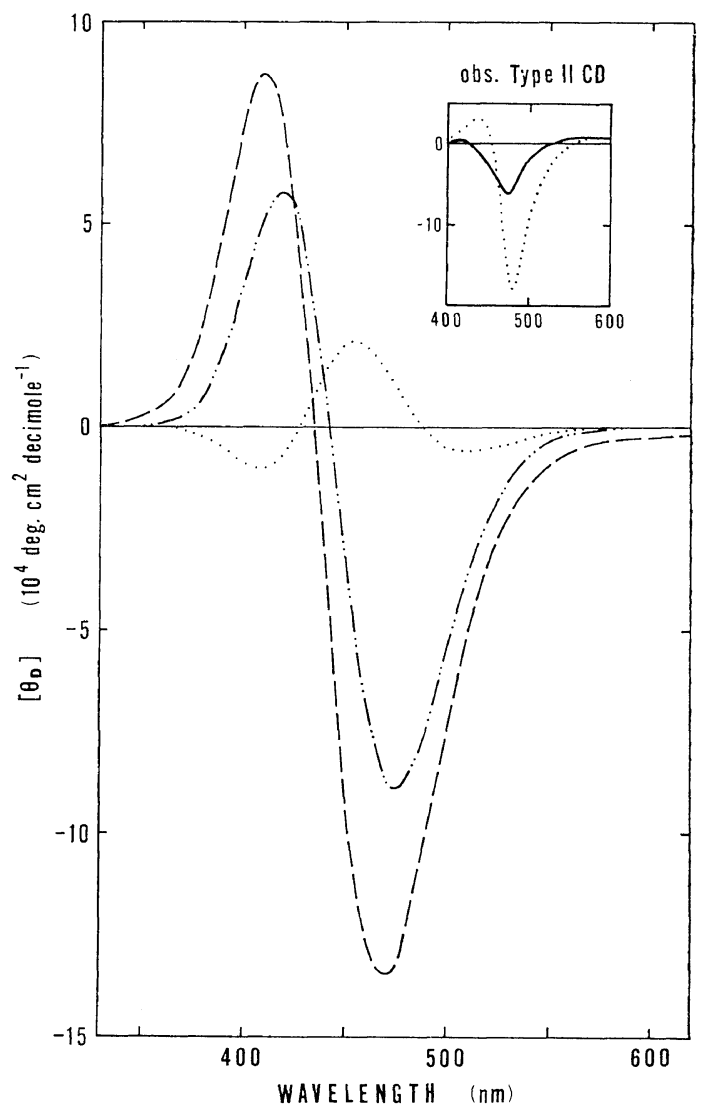

Figure 2. $C D$ of the ${ }^{1} L_{b}$ transition calculated for dimeric dye bound to polypeptides generated from the various regions: $\cdots$, region $1 ;-\cdots-$, region $\beta_{2} \alpha_{1}\left(P_{\alpha}=0.57\right) ;-\cdots$, region $\alpha_{1} ; \quad M=50, \quad N=10$, $\theta_{1}=135^{\circ} ; \phi_{1}=0^{\circ}, \theta_{\mathrm{s}}=90^{\circ}, \phi_{\mathrm{s}}=90^{\circ}$, and $l_{\mathrm{c}}=5.0 \AA$. Insert: - the observed $\mathrm{CD}$ for the acridine orange- $\operatorname{poly}(\boldsymbol{S}$-carboxymethyl-L-cysteine) system of $[\mathrm{P}] /[\mathrm{D}] 2$ at $[\mathrm{D}]=4.94 \times 10^{-5} M$ and $\mathrm{pH} 6.81^{1}$; $\cdots$, the observed $\mathrm{CD}$ for the acridine orangelow-molecular-weight poly( $S$-carboxymethyl-L-cysteine) (E515) system of $[\mathrm{P}] /[\mathrm{D}] 167$ at $[\mathrm{D}]=5.2 \times$ $10^{-5} M$ and $\mathrm{pH} 4.71 .^{2}$ 
in Table II. As predicted and shown practically, the steric hindrance of bound dyes occurs very frequently on the more compact polypeptide chain conformations and also more for $\theta_{1}=90^{\circ}$ than for $\theta_{1}=0^{\circ}$.

The zeroth-order $\mathrm{CD}$ for the ${ }^{1} \mathrm{~L}_{\mathrm{b}}$ transition of dimeric dye bound to a polypeptide was evaluated by eq 1 to 3 and yielded a conservative pair of ellipticity bands, the rotatory strengths of which sum to zero. The first-order nonconservative calculation for the ${ }^{1} \mathrm{~L}_{\mathrm{b}}$ transition arising from the interaction with the ${ }^{1} \mathbf{B}_{\mathrm{b}}$ transition was carried out by means of eq 5 and 6 . Figure 1 shows that, for the $\alpha_{1}$ region generating the more compact polypeptide chains, the angle $\theta_{1}=90-135^{\circ}$ results in a conservative pair of $C D$ bands with a negative band around 500 to $470 \mathrm{~nm}$ and a positive band around 420 to 410 $\mathrm{nm}$, accompanied by a negative nonconservative band. The calculated results at $\theta_{1}=135^{\circ}$ for some regions are given in Figure 2. The $\alpha_{1}$ and $\beta_{2} \alpha_{1}\left(P_{\alpha}=0.57\right)$ regions yield a pair of CD bands with the same sign as the Type II CD, while the other four regions cannot reproduce

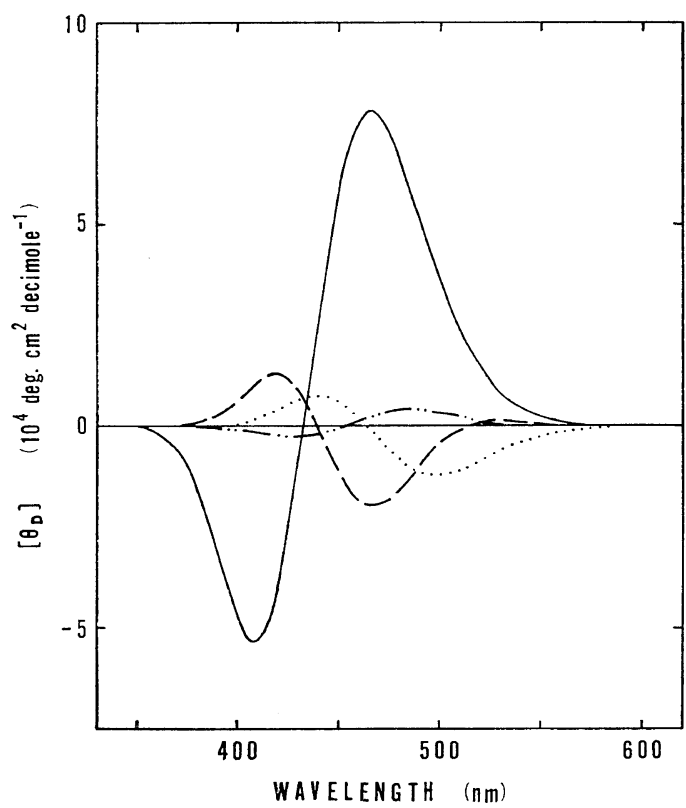

Figure 3. The $\theta_{1}$ dependence of the $C D$ of the ${ }^{1} L_{b}$ transition calculated for dimeric dye bound to polypeptides generated from the $\beta_{3}$ region: ,$- 0^{\circ} ; \cdots, 45^{\circ} ;-\cdots-, 90^{\circ} ;---, 135^{\circ} ; M=$ 50, $N=10, \phi_{1}=0^{\circ}, \theta_{\mathrm{s}}=90^{\circ}, \phi_{\mathrm{s}}=90^{\circ}$, and $l_{\mathrm{c}}=5.0 \AA$. the Type II CD.

For the $\beta_{3}$ region generating more extended polypeptide chains, $\theta_{1}=0^{\circ}$ gives rise to a pair of $C D$ bands, having a positive band at the longer wavelength side, and a positive nonconservative $C D$, as seen in Figure 3. The CD bands calculated at $\theta_{1}=0^{\circ}$ for the regions other than the 1 region are also consistent, in their sign and position, with the Type III CD, as well as the $\beta_{3}$ region. This is shown in Figure 4. However, among them, the $\alpha_{1}$ and $\beta_{2} \alpha_{1}\left(P_{\alpha}=0.57\right)$ regions are not likely for the Type III model, because they generate compact polypeptide chains.

The calculation assuming the bound species to be monomeric dye was also carried out and the results are shown in Figures 5 and 6 . Figure

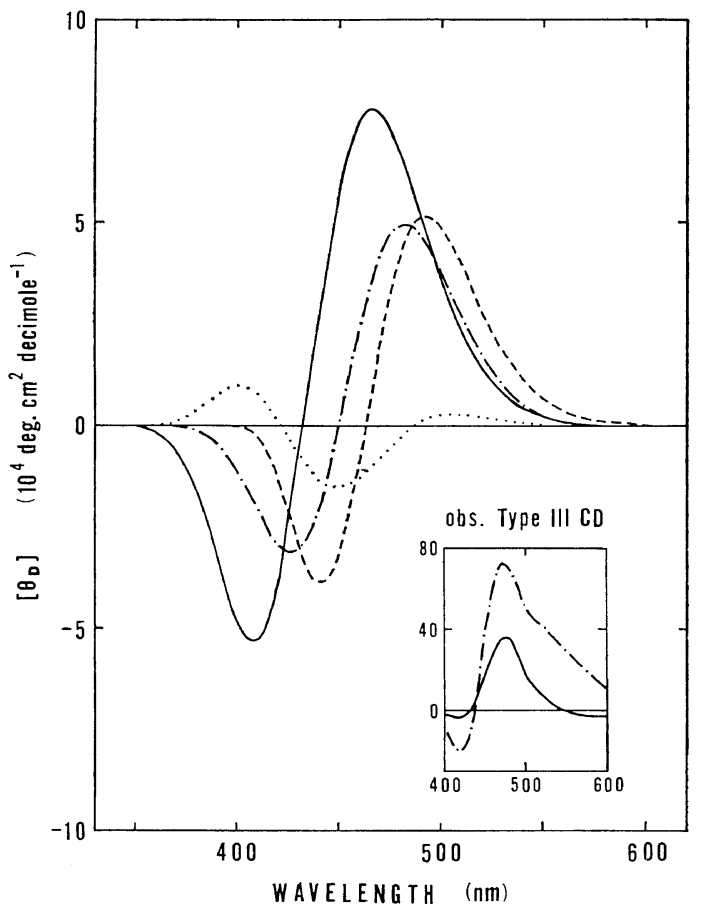

Figure 4. $C D$ of the ${ }^{1} \mathrm{~L}_{b}$ transition calculated for dimeric dye bound to polypeptides generated from the various regions: - , region $\beta_{3}$; -..., region 2 ; - - - region $\beta_{2} \alpha_{1}\left(P_{\alpha}=0.21\right) ; \cdots$, region 1 ; $M=50, \quad N=10, \quad \theta_{1}=0^{\circ}, \quad \phi_{1}=0^{\circ}, \theta_{\mathrm{s}}=90^{\circ}, \quad \phi_{\mathrm{s}}=90^{\circ}$, and $l_{\mathrm{c}}=5.0 \AA$. Insert: - $\longrightarrow$, the observed $C D$ for the acridine orange-poly $(S$-carboxymethyl-L-cysteine) system of $[\mathrm{P}] /[\mathrm{D}] 2$ at $[\mathrm{D}]=4.94 \times 10^{-5} M$

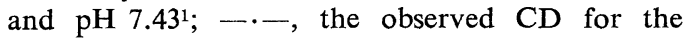
acridine orange-poly(L-glutamic acid) system of $[\mathrm{P}] /[\mathrm{D}] 1$ at $[\mathrm{D}]=2 \times 10^{-5} \mathrm{M}$ and $\mathrm{pH} 9.1 .^{4}$ 
5 gives the calculated $\mathrm{CD}$ at $\theta_{1}=135^{\circ}$ for the $\alpha_{1}$ and $\beta_{2} \alpha_{1}\left(P_{\alpha}=0.57\right)$ regions. $\mathrm{A}$ negative band around 520 to $505 \mathrm{~nm}$ and a positive one around 450 to $435 \mathrm{~nm}$ appear as a conservative $\mathrm{CD}$, together with a negative nonconservative band. The signs of the bands are found to be consistent with the Type $\mathrm{II}^{\prime} \mathrm{CD}$. The calculation at $\theta_{1}=0^{\circ}$ for the $\beta_{3}, \beta_{2} \alpha_{1}\left(P_{\alpha}=0.21\right)$ and 2 regions shown in Figure 6 gives rise to a positive band

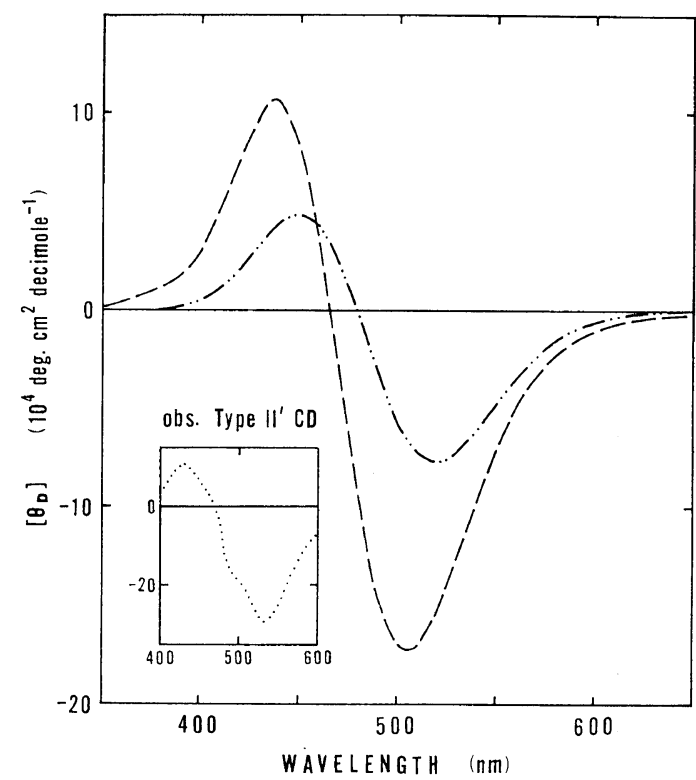

Figure 5. $C D$ of the ${ }^{1} L_{b}$ transition calculated for monomeric dye bound to polypeptides generated from the various regions: - - - region $\beta_{2} \alpha_{1}$ $\left(P_{\alpha}=0.57\right) ;---$, region $\alpha_{1} ; M=50, N=10, \theta_{1}=$ $135^{\circ}, \phi_{1}=0^{\circ}, \theta_{\mathrm{s}}=90^{\circ}, \phi_{\mathrm{s}}=90^{\circ}$, and $l_{\mathrm{c}}=5.0 \AA$. Insert: $\ldots$, the observed $\mathrm{CD}$ for the acridine orangelow-molecular-weight poly( $S$-carboxymethyl-L-cysteine) (E515) system of $[\mathrm{P}] /[\mathrm{D}] 0.8$ at $[\mathrm{D}]=4.94 \times$ $10^{-5} M$ and $\mathrm{pH} 4.84 .^{1}$ in the longer wavelength side and a negative band in the shorter wavelength side. The nonconservative $\mathrm{CD}$ is positive. The signs of bands are in agreement with those observed in the Type III' CD.

The nonconservative $C D$ of the ${ }^{1} L_{b}$ transition, arising from the interaction with the other

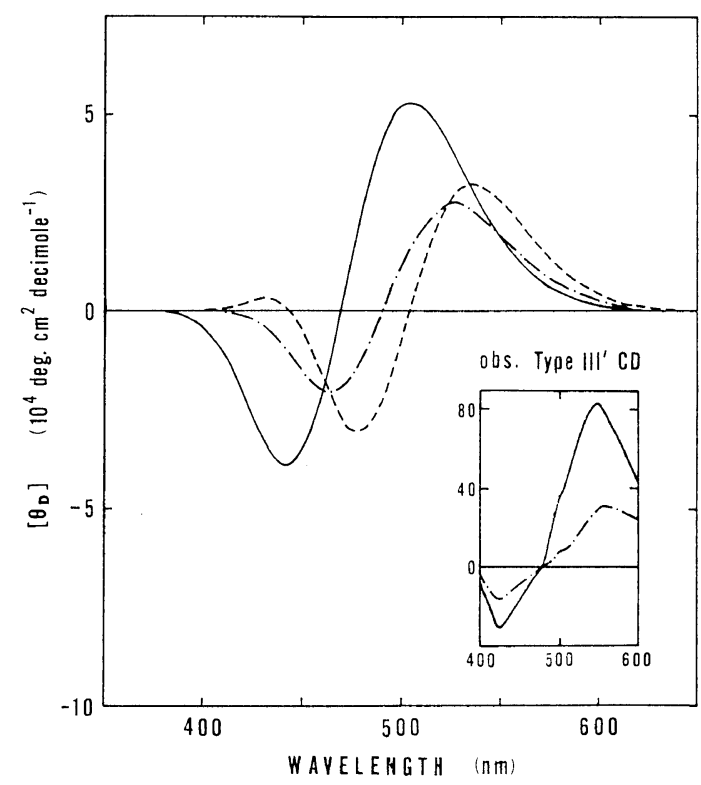

Figure 6. $\mathrm{CD}$ of the ${ }^{1} \mathrm{~L}_{\mathrm{b}}$ transition calculated for monomeric dye bound to polypeptides generated from the various regions: - - , region $\beta_{3}$; ----, region $2 ;-\cdot-, \quad \beta_{2} \alpha_{1}\left(P_{\alpha}=0.21\right) ; \quad M=50, \quad N=10$, $\theta_{\mathrm{l}}=0^{\circ}, \phi_{1}=0^{\circ}, \quad \theta_{\mathrm{s}}=90^{\circ}, \dot{\phi}_{\mathrm{s}}=90^{\circ}$, and $l_{\mathrm{c}}=5.0 \AA$. Insert: orange-poly $(S$-carboxymethyl-L-cysteine) system of $[\mathrm{P}] /[\mathrm{D}] 0.9$ at $[\mathrm{D}]=4.94 \times 10^{-5} \mathrm{M}$ and $\mathrm{pH} 6.26 ;^{1}$ -.-, the observed $\mathrm{CD}$ for the acridine orangepoly(L-glutamic acid) system of $[\mathrm{P}] /[\mathrm{D}] 0.8$ at $[\mathrm{D}]=$ $4.94 \times 10^{-5} M$ and $\mathrm{pH} 7.00 .^{5}$

Table III. The calculated $\mathrm{CD}$ for the ${ }^{1} \mathrm{~L}_{\mathrm{b}}$ transition of dimeric dyes bound to random-coil polypeptides $\left(M=50, N=10, \phi_{1}=0^{\circ}, \theta_{\mathrm{s}}=90^{\circ}, \phi_{\mathrm{s}}=90^{\circ}\right.$, and $l_{\mathrm{c}}=5.0 \AA$ )

\begin{tabular}{|c|c|c|c|c|c|c|c|}
\hline \multirow{3}{*}{ Region } & \multirow{3}{*}{$\theta_{1}$, deg } & \multicolumn{2}{|c|}{ Conservative CD } & \multicolumn{4}{|c|}{ Nonconservative CD } \\
\hline & & \multirow{2}{*}{$\begin{array}{l}c / \nu \\
\mathrm{nm}\end{array}$} & \multirow{2}{*}[\theta]{$_{\mathrm{E}}$} & \multirow{2}{*}{$\begin{array}{l}c / \nu \\
\mathrm{nm}\end{array}$} & \multicolumn{3}{|c|}{$[\theta]_{\mathrm{E}}$} \\
\hline & & & & & ${ }^{1} \mathbf{B}_{\mathrm{b}}$ & ${ }^{1} \mathbf{L}_{\mathrm{a}}$ & ${ }^{1} \mathbf{B}_{\mathrm{a}}$ \\
\hline \multirow{2}{*}{$\alpha_{1}$} & \multirow{2}{*}{90} & 440 & 38,300 & \multirow[t]{2}{*}{455} & \multirow{2}{*}{$-32,200$} & \multirow{2}{*}{$-1,500$} & \multirow[t]{2}{*}{3,100} \\
\hline & & 505 & $-38,700$ & & & & \\
\hline \multirow{2}{*}{$\beta_{3}$} & \multirow{2}{*}{0} & 410 & $-56,700$ & \multirow[t]{2}{*}{455} & \multirow[t]{2}{*}{30,800} & \multirow[t]{2}{*}{700} & \multirow[t]{2}{*}{$-1,500$} \\
\hline & & 470 & 49,600 & & & & \\
\hline
\end{tabular}


electrically allowed transitions besides the ${ }^{1} \mathbf{B}_{\mathrm{b}}$ state, was obtained according to eq 4 to 6 . The results for the two systems of the $\alpha_{1}$ region at $\theta_{1}=90^{\circ}$ and of the $\beta_{3}$ region at $\theta_{1}=0^{\circ}$ are given in Table III. The contribution from the ${ }^{1} \mathrm{~L}_{\mathrm{a}}$ and ${ }^{1} \mathbf{B}_{\mathrm{a}}$ states was very small, as predicted from their small dipole moments, in comparison with that from the ${ }^{1} B_{b}$ state.

The dependence of calculated $C D$ values on the parameter $N$ was examined for the $\beta_{3}$ region at $\theta_{1}=0^{\circ}$. For the Type III complex, the sequence of chromophores can be longer. However, the calculated molar ellipticity at $N=20$ was not large as compared with that at $N=10$, because the available number $N^{\prime}$ of chromophores decreased owing to steric hindrance.

We have given $C D$ values averaged over $M=50$. In some cases, convergence was not always reached, but these should not be far from their asymptotes.

The ${ }^{1} L_{\mathrm{a}}$ Transition of Acridine Orange Bound to Random-Coil Polypeptide Chain

In the visible region, there is an electronic transition of the ${ }^{1} L_{a}$ state of acridine orange, besides ${ }^{1} \mathrm{~L}_{\mathrm{b}}$ state. In order to explain the additional $\mathrm{CD}$ bands observed at the longer wavelength side than the ${ }^{1} \mathrm{~L}_{\mathrm{b}}$ bands in Types II and III $C D,{ }^{1}$ the $C D$ of the ${ }^{1} L_{a}$ transition should be considered. As the dipole moment of the ${ }^{1} \mathrm{~L}_{\mathrm{a}}$ transition is very small, conservative rotational strength should be small, according to eq 1 . However, from eq 4 , the nonconservative term caused by the interaction with the ${ }^{1} \mathrm{~L}_{\mathrm{b}}$ transition may have a significant magnitude, because the unperturbed frequency of the ${ }^{1} \mathrm{~L}_{\mathrm{a}}$ state at $540 \mathrm{~nm}$ is close to that of ${ }^{1} \mathrm{~L}_{\mathrm{b}}$ at $458 \mathrm{~nm}$.

For the two typical cases of dimeric dyerandom-coil polypeptide model, the nonconservative $C D$ of the ${ }^{1} L_{a}$ transition due to the interaction with the other transitions was evaluated by the eq 4 and 5 , and these results are shown in Table IV. The shorter axis of dye molecular plane was tilted from $\theta_{\mathrm{s}}=90^{\circ}$ and $\phi_{\mathrm{s}}=90^{\circ}$. For the $\alpha_{1}$ region at $\theta_{1}=90^{\circ}$, the nonconservative $C D$ by interaction with the ${ }^{1} \mathrm{~L}_{\mathrm{b}}$ transition was positive for the tilt angle $\theta_{\mathrm{s}}=90$ $135^{\circ}$. For the $\beta_{3}$ region at $\theta_{1}=0^{\circ}$, it was negative for the tilt angle $\phi_{\mathrm{s}}=90-135^{\circ}$. These signs are coincident with the observed additional band at the longest wavelength side, apart from their calculated smaller magnitudes. The contributions from the ${ }^{1} B_{a}$ and ${ }^{1} B_{b}$ transitions are smaller than those from the ${ }^{1} \mathrm{~L}_{\mathrm{b}}$ transition, as would be expected.

\section{DISCUSSION}

Myer and Foss ${ }^{22}$ and Eyring, et al. ${ }^{23}$ reported that the Cotton effect in the visible region was induced for acridine orange-poly(L-glutamic acid) complex even under a condition where the structure of poly(L-glutamic acid) is a randomcoil. Sato and Hatano ${ }^{4}$ claimed that, in the cases where $[\mathrm{P}] /[\mathrm{D}]$ is around unity, a complex having an ordered structure can be formed even in the neutral and alkaline $\mathrm{pH}$ region and proposed a binding model for Type III CD. In

Table IV. The nonconservative contributions for the ${ }^{1} \mathrm{~L}_{\mathrm{a}}$ transition of dimeric dyes bound to random-coil polypeptides $\left(M=50, N=10\right.$, and $l_{\mathrm{c}}=5.0 \AA$ )

\begin{tabular}{|c|c|c|c|c|c|c|c|c|c|c|}
\hline \multirow[t]{2}{*}{ Region } & \multicolumn{2}{|c|}{$\begin{array}{l}\text { Longer } \\
\text { axis }\end{array}$} & \multicolumn{2}{|c|}{$\begin{array}{l}\text { Shorter } \\
\text { axis }\end{array}$} & \multicolumn{3}{|c|}{$\begin{array}{l}\text { Rotatory strength, } \\
10^{-38} \mathrm{erg} \cdot \mathrm{cm}^{3}\end{array}$} & \multicolumn{3}{|c|}{$\begin{array}{c}\text { CD, } \\
10^{4} \mathrm{deg} \cdot \mathrm{cm}^{2} \text { decimol }^{-1}\end{array}$} \\
\hline & $\theta_{1}$ & $\phi_{1}$ & $\theta_{\mathrm{s}}$ & $\phi_{\mathrm{s}}$ & ${ }^{1} \mathrm{~L}_{\mathrm{b}}$ & ${ }^{1} \mathbf{B}_{\mathrm{a}}$ & ${ }^{1} \mathbf{B}_{\mathrm{b}}$ & ${ }^{1} \mathbf{L}_{\mathrm{b}}$ & ${ }^{1} \mathbf{B}_{\mathrm{a}}$ & ${ }^{1} \mathbf{B}_{\mathrm{b}}$ \\
\hline \multirow{4}{*}{$\alpha_{1}$} & \multirow{4}{*}{90} & \multirow{4}{*}{0} & 0 & 90 & -0.0166 & 0.0008 & -0.0044 & -723 & 33 & -189 \\
\hline & & & 45 & 90 & -0.0516 & 0.0011 & -0.0135 & -2245 & 47 & -589 \\
\hline & & & 90 & 90 & 0.0288 & 0.0002 & 0.0076 & 1252 & 9 & 328 \\
\hline & & & 135 & 90 & 0.0602 & -0.0009 & 0.0158 & 2619 & -37 & 687 \\
\hline \multirow{4}{*}{$\beta_{3}$} & \multirow{4}{*}{0} & \multirow{4}{*}{0} & 90 & 0 & 0.0002 & -0.0000 & 0.0000 & 7 & 0 & 2 \\
\hline & & & 90 & 45 & 0.0044 & -0.0003 & 0.0012 & 190 & -11 & 50 \\
\hline & & & 90 & 90 & -0.0140 & 0.0002 & -0.0037 & -607 & 6 & -159 \\
\hline & & & 90 & 130 & -0.0242 & 0.0003 & -0.0064 & $-1,054$ & 11 & -276 \\
\hline
\end{tabular}


consideration of the induced $C D$ designated as Types II, III, II', and III' $^{\prime}$, however, we can say that a polymer in dye-polymer complex remains the random-coil form, on the basis of our experimental evidence. ${ }^{1}$ Incidentally, Salter, et al. ${ }^{6}$ supposed that there was no evidence to support a completely helical structure for the glycosaminoglycan, when it adsorbs dyes and induces the so-called Types II and III CD.

The observed CD spectra ${ }^{1}$ of Types II, III, $\mathrm{II}^{\prime}$, and III' $^{\prime}$ are shown in the inserts of Figures $2,4,5$, and 6 , and are compared with one calculated. A pair of ${ }^{1} L_{b}$ bands in the visible region of Types II and II $^{\prime}$ CD were successfully obtained by the CD calculated for the binding model, where dyes are bound to the more compact polypeptide chain generated from the $\alpha_{1}$ and/or $\beta_{2} \alpha_{1}\left(P_{\alpha}=0.57\right)$ region and the longer axes of dyes are arranged on it with a tilt of $\theta_{1}=$ $90-135^{\circ}$; that is, they are tilted slightly from the direction along the polypeptide chain. A pair of ${ }^{1} \mathrm{~L}_{\mathrm{b}}$ bands of Types III and III' CD were reproduced by a binding model of dye on the more extended polypeptide chain generated from the $\beta_{3}, 2$ and/or $\beta_{2} \alpha_{1}\left(P_{\alpha}=0.21\right)$ region, where the longer axes of dye have a tilt of $\theta_{1}=0^{\circ}$; i.e., they were almost perpendicular to the polymer chain.

The binding of the dye to the polymer is

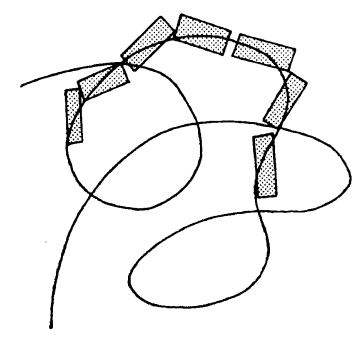

(a)

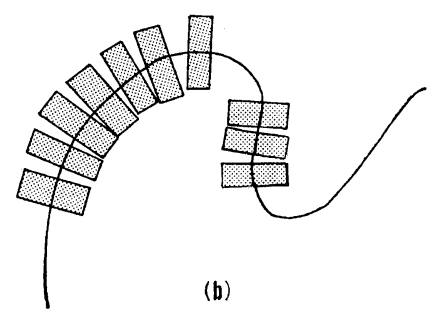

Figure 7. Two models proposed for the acridine orange-random-coil polypeptide complex. primarily electrostatic; one of the nitrogen atoms of the dye binds to an ionized carboxyl group of the polymer. In order to fix the dye rigidly on the polymer, either of the other nitrogen atoms must approach the other carboxyl groups to form a hydrogen bond. Then the longer axis of the molecular plane of dye should favorably lie along the polymer chain, as illustrated in Figure 7(a). This is considered as a model for Types II and II' complexes. For the more extended random-coil polymer, it should be possible for dyes bound to nearby residues to stack together and hence the molecular planes of the dye become almost perpendicular to the polypeptide chain, as shown in Figure $7(b)$. This is a model of Types III and III' $^{\prime}$ complexes.

As the observed $C D$ is more nonconservative than the calculated $\mathrm{CD}$ based on the conservative ${ }^{1} \mathrm{~L}_{\mathrm{b}}$ and its nonconservative coupling with ${ }^{1} \mathbf{B}_{\mathrm{b}}$, other contributions must be taken into consideration for calculation. However, consideration of the nonconservative $C D$ arising from the coupling with the ${ }^{1} L_{a}$ or ${ }^{1} B_{a}$ state brought about merely a small increase in the magnitude of the ${ }^{1} \mathrm{~L}_{\mathrm{b}}$ bands and was not sufficient to reproduce the observed nonconservative values.

Types II and III CD have additional bands at the longer wavelength side, besides a pair of ${ }^{1} L_{b}$ bands, in three ways; ${ }^{1}$ one has a broad and weak band around 600 to $540 \mathrm{~nm}$, one has a shoulder around 540 to $510 \mathrm{~nm}$ and one has both of them. Their difference seems to originate from the intensity of both bands, because the two additional bands have opposite signs to each other. The band around 540 to $510 \mathrm{~nm}$ may be assigned to the contamination of Type $\mathrm{II}^{\prime}$ or III $^{\prime} \mathrm{CD}$, because this band grows into a strong band around 600 to $540 \mathrm{~nm}$ of Type II' $^{\prime}$ or III' $\mathrm{CD}$ with changing the $\mathrm{pH} .{ }^{1} \quad$ The ${ }^{1} \mathrm{~L}_{\mathrm{a}}$ transition can be assigned to the band around 600 to 540 $\mathrm{nm}$. The calculated nonconservative $\mathrm{CD}$ for the ${ }^{1} L_{a}$ transition was too small in magnitude to coincide with the observed ones.

The observed molar ellipticity for Types III and III' $^{\prime} \mathrm{CD}$ is larger than one calculated. It very much increases beyond the magnitude of CD drawn in Figures 2, 4, 5, and 6, when the $[\mathrm{P}] /[\mathrm{D}]$ value decreases. ${ }^{1}$ Thus, the deviation from the calculated $\mathrm{CD}$ becomes larger. A change in value of parameter to $N=20$ does not 
lessen the disagreement above. In order to reduce the discrepancy in our calculation, we will have to consider some theoretical improvement of the CD calculation. Salter, et al., ${ }^{6}$ reported that the aggregate of acridine orangeglycosaminoglycan complexes exhibits $C D$ bands with large magnitude, and designated as Type II or III. If their interpretation is correct, the existence of a long-range ordering of dyepolymer complexes within the aggregate must be taken into account for our calculation.

The sign of the $C D$ bands induced on the dye bound to the polymer having a regular structure can be determined mainly by the sign of the strongest interaction potential between the pair of chromophores. ${ }^{2,3,5}$ However, in the dyerandom-coil polymer complex, this is not the case. The sign of most of interaction potentials was found to be negative (attractive) at $\theta_{1}=90^{\circ}$ for all the regions. Nevertheless, the sign of $\mathrm{CD}$ bands is not definite but depends on a dimension of the polypeptide, extended or compact.

Our method of calculation was similar to that by Zubkov, et al. ${ }^{9}$ We adopted the regions of rotational angles $\varphi$ and $\psi$ which Zubkov, et al. used for the calculation of the amide $\pi-\pi^{*} \mathrm{CD}$ of random-coil polypeptide. It may be noted that the visible $C D$ of dye-random-coil polymer complex is very sensitive to the selection of the region, while the amide $\pi-\pi^{*} \mathrm{CD}$ changes only in the magnitude at every region, even when the selected region differs. For the CD calculation of $\pi-\pi^{*}$ transition of random-coil polypeptide, the nearest neighbor approximation holds well, while the CD calculation for the transition in the visible region of acridineorange-random-coil polymer mixture can not be carried out by the nearest neighbor approximation, as expected from the chromophore position far away from the main chain and as elucidated by the calculated potential values.

Acknowledgment. The author wishes to express the sincere appreciation to Professor S. Ikeda of Nagoya University for making many useful suggestions and for his carefully reading the manuscript.

\section{APPENDIX}

Amide $\pi-\pi^{*}$ Transition of Random-Coil Poly-

\section{peptide}

The calculation of the $C D$ associated with amide $\pi-\pi^{*}$ transition of random coil polypeptide was carried out by eq 1,3 , and 7 . When the monopole approximation is used in representing the $\pi-\pi^{*}$ electric transition moment, the dipole moment $\boldsymbol{\mu}_{k 0 \mathrm{e}}$ is given by $\boldsymbol{\mu}_{k 0 \mathrm{c}}=$ $\sum_{\sigma} \rho_{k 0 \mathrm{e}, \boldsymbol{\sigma}} \boldsymbol{r}_{k \sigma}$, where $\rho_{k 0 \mathrm{e}, \sigma}$ is the monopole charge associated with the transition $0-\mathrm{e}$ at the position $\sigma$ in the $k$-th residue and $\boldsymbol{r}_{k \sigma}$ is the vector of the position $\sigma . \quad \boldsymbol{r}_{k}$ is taken to be the center of the transition monopole charges. The interaction potential in the monopole approximation is

$$
V_{l k 0 \mathrm{e}, l 0 \mathrm{e}}=\sum_{\sigma} \sum_{\tau} \rho_{k 0 \mathrm{e}, \sigma} \rho_{l 0 \mathrm{e}, \tau}\left(\boldsymbol{r}_{k \sigma, l \tau} \cdot \boldsymbol{r}_{k \sigma, l \tau}\right)^{-1 / 2} \text { (A.1) }
$$

where $V_{k 0 \mathrm{e}, k 0 \mathrm{e}}=0 . \quad \boldsymbol{r}_{k \sigma, l \tau}$ is the distance vector between the position $\sigma$ in the $k$-th residue and the position $\tau$ in the $l$-th one. It is written by eq 7 , in which $\boldsymbol{r}_{k}$ is replaced by $\boldsymbol{r}_{k \sigma}$ and $\boldsymbol{r}_{l}$ by $\boldsymbol{r}_{l \tau}$. The values of the monopole parameter and the other optical parameters were adopted from Tonoco, et al. ${ }^{24,25}$

The CD calculation for the amide $\pi-\pi^{*}$ transition of $\alpha$-helical polypeptide was done with $\varphi=122^{\circ}$ and $\psi=133^{\circ} .{ }^{18}$ The calculated CD for $N=20$ were found to agree fairly well with those computed by the helical parameter method. ${ }^{5}$

\section{REFERENCES}

1. T. Imae and S. Ikeda, Biopolymers, 14, 1213 (1975).

2. S. Ikeda and T. Imae, Biopolymers, 10, 1743 (1971).

3. S. Ikeda and T. Imae, Polym. J., 4, 301 (1973).

4. Y. Sato and M. Hatano, Bull. Chem. Soc. Jpn., 46, 3339 (1973).

5. T. Imae and S. Ikeda, Biopolymers, 15, 1655 (1976).

6. M. K. Salter, E.W. Abrahamson, and W. B. Rippon, Biopolymers, 15, 1251 (1976).

7. A. E. Tonelli, Macromolecules, 2, 635 (1969).

8. D. Aebersold and E. S. Pysh, J. Chem. Phys., 53, 2156 (1970).

9. V. A. Zubkov, T. M. Birshtein, I. S. Milevskaya, and M. V. Volkenstein, Biopolymers, 10, 2051 (1971).

10. E. W. Ronish and S. Krimm, Biopolymers, 11, 1919 (1972).

11. W. Moffitt, D. Fitts, and J. Kirkwood, Proc. 
Natl. Acad. Sci. U.S., 43, 723 (1957).

12. I. Tinoco, Adv. Chem. Phys., 4, 113 (1962).

13. V. Zanker, M. Held, and H. Ramensee, $Z$. Naturforsch., 14b, 789 (1959).

14. V. Zanker, Z. phys. Chem., 2, 52 (1954).

15. V. Zanker, Z. phys. Chem., 199, 225 (1952).

16. A. Wittwer and V. Zanker, Z. phys. Chem., Neue Folge, 22, 417 (1959).

17. J. R. Platt, J. Chem. Phys., 17, 484 (1949).

18. P. J. Flory, Statistical Mechanics of Chain Molecules, Interscience, New York, N.Y., 1969.

19. S. Arnott, S. D. Dover, and A. Elliot, J. Mol.
Biol., 30, 201 (1967).

20. D. A. Brant, W. G. Miller and P. J. Flory, $J$. Mol. Biol., 23, 57 (1967).

21. Y. P. Myer, Macromolecules, 2, 624 (1969).

22. B. C. Myhr and J. G. Foss, Biopolymers, 4, 949 (1966).

23. E. J. Eyring, H. Kraus, and J. T. Yang, Biopolymers, 6, 703 (1968).

24. I. Tinoco, Jr., R. W. Woody, and D. F. Bradley, J. Chem. Phys., 38, 1317 (1963).

25. R. W. Woody and I. Tinoco, Jr., J. Chem. Phys., 46, 4927 (1967). 